\title{
Three- to Five-Dimensional Biomedical Multisensor Imaging for the Assessment of Neurological (Dys)function
}

\author{
Luc M. Bidaut, Roberto Pascual-Marqui, Jacqueline Delavelle, Alain Naimi, Margitta Seeck, \\ Christophe Michel, Daniel Slosman, Osman Ratib, Daniel Ruefenacht, Theodor Landis, \\ Nicolas de Tribolet, Jean-Raoul Scherrer, and François Terrier
}

\begin{abstract}
This report describes techniques and protocols implemented at the Geneva Canton University Hospitals (HUG) for the combination of various biomedical imaging modalities and sensors including electromagnetic tomography, to study, assess, and localize neurological (dys)function. The interest for this combination stems from the broad variety of information brought out by (functional) magnetic resonance imaging, magnetic resonance spectroscopy, computed tomography, single-photon emission tomography, positron emission tomography, and electromagnetic tomography. Combining these data allows morphology, metabolism, and function to be studied simultaneously, the complementary nature of the information from these modalities becoming evident when studying pathologies reflected by metabolic or electrophysiologic dysfunctions. Compared with other current multimodality approaches, the one at the HUG is totally compatible with both clinical and research protocols, and efficiently addresses the multidimensional registration and visualization issues. It also smoothly integrates electrophysiology and related data as fully featured modalities.
\end{abstract}

Copyright $\odot 1996$ by W.B. Saunders Company

KEY WORDS: medical imaging (MI), multimodality (MM) MI, multisensor (MS) MI, multidimensional MI, three-dimensional MI, brain function, epilepsy, computers, radiology.

$\mathbf{T}$ HE WORLD of biomedical imaging can be divided in two major tracks: one morphological, where the images show the underlying anatomic structures; the other metabolic or functional, where the images produced provide information about the metabolism or the function of the underlying tissues. The first track includes $\mathrm{x}$-ray computed tomography (CT) and magnetic resonance imaging (MRI). The second track includes single-photon emission computed tomography (SPECT), positron-emission tomography (PET), magnetic resonance spectroscopy (MRS), and functional MRI (fMRI).

The two tracks provide information that has clearly been recognized as complementary and may consequently often benefit from direct comparison in various protocols or pathologies. For example, multimodality (MM) approaches are now well accepted in oncology, either for the brain or other tissues. ${ }^{1.5}$ Beyond tumors, studies are also under way to investigate brain function through MM approaches, ${ }^{6,7}$ and other pathologies or organs may also benefit from such approaches. ${ }^{8-11}$

As the modalities making up the two tracks include both direct imaging and pseudoimaging modalities (eg, parametric imaging), a more generic name had to be coined and was chosen as "sensor" by analogy with space- and airbased remote sensing imaging concepts. In the rest of this report, modality and sensor are considered to have rather similar meanings, the latter being considered to also include nondirect imaging "modalities."

Because of the spreading need for multimodality/multisensor (MS) approaches, and since most modalities/sensors are still not produced on the same machine or in the same spatial referential, many groups have been involved in three-dimensional (3D) data sets registration issues so as to be able to work with different modalities in a common space. ${ }^{12}$ Approaches used for registering data sets can either be prospective or retrospective. Prospective approaches often imply artificial markers or fiducials placed on the patient before the acquisitions. In these approaches, the markers, not the actual anatomy, are being aligned by the registration process. Retrospective techniques can make use of routinely acquired data and, ideally, do not imply a priori knowledge about any subsequent MM alignment. Data sets are acquired normally, without additional locator devices. Most often, similar anatomic structures are extracted from the initial data sets, and their 3D distance is minimized by iterative manipulation of the $3 \mathrm{D}$ interset geometry transformation

From the Departments of Medical Informatics, Neurology, Radiology, Nuclear Medicine, and Neurosurgery, Geneva Canton University Hospital, Geneva, Switzerland.

Address reprint requests to Luc Bidaut, PhD, UIN-DIMRadiology, Geneva Canton University Hospital, 24 Rue Michelidu-Crest, CH-1211 Geneva 14, Switzerland.

Copyright $\odot 1996$ by W.B. Saunders Company

0897-1889/96/0904-0005\$3.00/0 
(eg, through 3D translations and rotations). The manipulation of the data sets to reach the optimal alignment transformation can range from direct manual interaction, to point-topoint correspondence, ${ }^{13,14}$ principal axes registration, ${ }^{15}$ 3D surfaces alignment, ${ }^{16-18}$ and also $3 \mathrm{D}$ volume correlation. ${ }^{19}$ None of these approaches is general enough to take the best care of every MM registration problem, and this is the reason why there is continuous development in this field. For example, different graphical primitives can be used simultaneously in the computation of the 3D distance between two data sets to improve the result of the registration. ${ }^{20}$ Other developments tend to accelerate the convergence of iterative methods by modifying the convergence engine with no major modification of the interset distance cost engine. ${ }^{19,21}$ Although the speed of convergence is certainly important for widespread acceptance and usage of the MM concepts, it is important to stress that it is the cost engine itself that makes the true identity of a registration approach.

In the scope of functional data acquisition and imaging of the brain, electromagnetic tomography (EMT), either based on electroencephalography (EEG) or magnetoencephalography (MEG), can be seen as a functional modality able to complement PET-SPECT and fMRI ideally. Compared with them, EMT, which does not necessarily require complex external devices (eg, when based on EEG), provides the actual capacity to follow-on an arbitrary time scale close to real time-the electrical changes caused in the brain by an external stimulus or an internal (dys)function. Besides the obvious advantages of EEG-based EMT (fast, relatively inexpensive, and no special requirements for acquisition set-up), this technique generally suffers from its intrinsic low spatial resolution. As such, it requires accurate matching and fusion with actual brain anatomy to be correctly interpreted and show its full potential for functional analysis.

Once properly registered with anatomy, the timing capabilities of EMT should eventually allow the anatomic path of a (dys)functional electromagnetic (EM) activity to be traced in near real time. In addition to the spatial localization aspect, and because of the totally new information they provide, registering the EMT maps to other modalities can also lead to a better understanding and assessment of the underlying functional and metabolic behavior of the tissues.

Most present modalities being tomographies, the multisensor space initially has three dimensions. For dynamic acquisitions, time adds a fourth dimension, and considering various modalities or sensors adds another one. The whole MS space ends up being a 5D one (Fig 1), which does not consider the possible postprocessing issues (pharmacokinetic modeling in PET, etc), which can also add parameter dimensions.
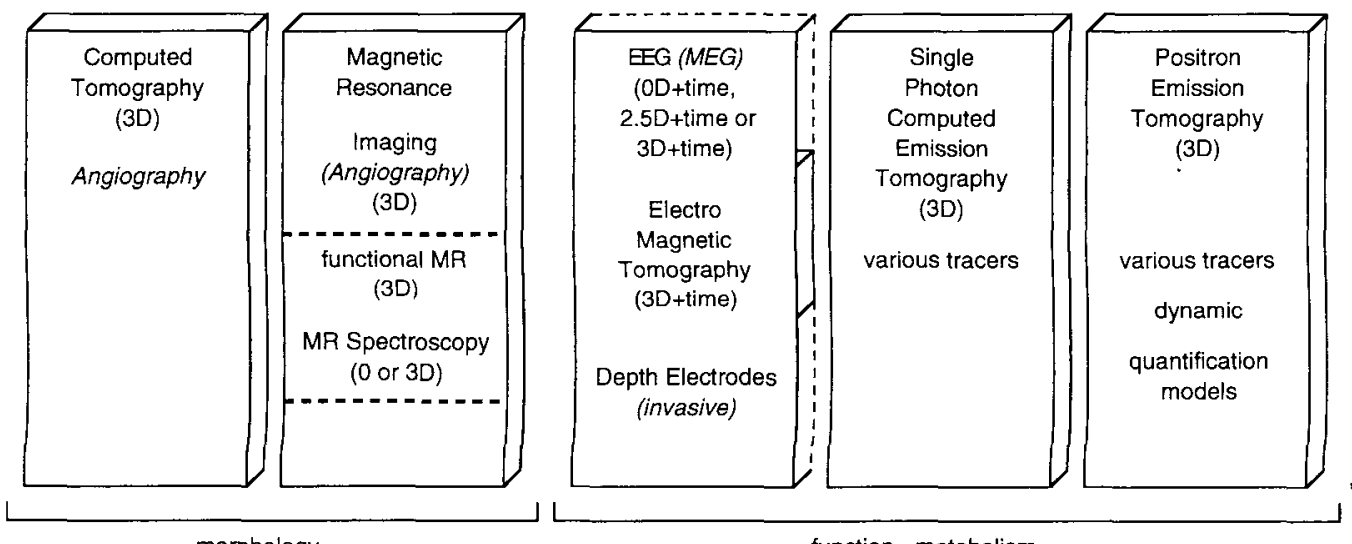

tomography $\rightarrow 3 D$

morphology

L

function - metabolism

modality - sensor $=5$ th $D$

(processing $\rightarrow$ parameters $=6$ thD $)$

5D MultiSensor space

Fig 1. 5D multisensor space 
The purpose of this report is to present the current multisensor environment and means that have been implemented in Geneva to register the different data sets and visualize them simultaneously. Compared with other approaches, the Geneva integrated multisensor imaging and processing system (IMIPS) has been developed to accommodate both clinical and research protocols and applications. EMT and EEG have also been integrated in IMIPS as fully featured modalities and can readily be compared with the other modalities both in space and time. Because of its rather new entry in the multisensor field, and because of the specificity it adds to IMIPS compared with other multisensor approaches, there will be some emphasis on the specific processing and use of EMT data within this context.

Typical examples of current presurgical assessment of pharmacoresistant epilepsy will be succinctly presented to illustrate the use and impact of IMIPS on actual clinical cases. For this specific pathology, better and more objective localization of the foci is obtained from MS approaches before possible surgical resection: the MR data can show possible morphological lesions and the interictal fluorodeoxy-Glucose (FDG)-PET should show localized hypometabolism ${ }^{22,23}$; the comparison between interictal and ictal hexamethyl-propylene-amineoxime (HMPAO) or ethylene-cysteinate-dimer (ECD)-SPECT should show an increase of perfusion, 23,24 and the EEG reconstructed during the ictus can show the EM evolution of the crisis in near real time. Most often, only a few of the individual modality clues are simultaneously visible for any single patient. The combination of data of various types is then expected to improve the localization of the triggers or potential epileptic foci from complementary viewpoints, and is, therefore, of great interest for the planning of possible surgical resection or further investigation, such as depth electrode implantation.

\section{MATERIALS AND METHODS}

\section{Acquisition and Processing Environments}

The medical imaging equipment available at the Geneva Canton University Hospitals (HUG) is a typical multivendor configuration.
MR: Picker Edge 1.5T

(Picker International, Inc, Highland Heights, $\mathrm{OH}$ )

CT: $\quad$ Picker PQ2000 spiral CT

(Picker International, Inc, Highland Heights, OH)

SPECT: Toshiba three-head prism

(Toshiba Medical Systems, Inc, Tustin, CA)

PET: $\quad$ CTI

(CTI, Inc, Knoxville, TN)

Siemens RPT (rotating positron tomograph)

ART (advanced rotating tomograph) rotating PET

(Siemens Medical Systems, Inc, Iselin, NJ)

The EEG acquisition is performed on commercial personal computer-based multichannel equipment. From the scalp electrodes' locations and EEG measurements, the current density vector field can be reconstructed in $3 \mathrm{D}$ and low resolution. For example, low resolution electromagnetic tomography (LORETA), ${ }^{25}$ which is used in Geneva, reconstructs the current density vector field (see Fig 4) by making various assumptions about the shape of the head (virtual sphere on which the electrodes lie), the conductivity inside the head (concentric brain, skull, and scalp spherical shells), and the continuity of the neuronal generators distribution within the brain.

All imaging devices are connected to a hospital-wide picture archiving and communication system (PACS) ${ }^{26} \mathrm{At}$ the present time, archiving of images is performed on a routine basis only for data from MR and CT equipment, whereas SPECT and PET data are converted and transferred only on demand.

Data retrieval, conversion, alignment, fusion, and realtime 3D visualization are all performed on a Silicon Graphics (Mountain View, CA) workstation (Crimson Reality Engine), mostly under Explorer (SGI-Numerical Algorithms Group, Oxford, UK), a module-based multiplatform software engineering system for which many specific modules had to be developed to fulfill the needs of multidimensional and MS biomedical imaging.

To comply with the requirements of the PACS, and also to make IMIPS truly compatible with clinical applications, all reference and resliced data sets produced in the scope of IMIPS are converted to the digital imaging and communication in medicine-compatible ${ }^{27}$ Papyrus file format and made globally available on the PACS for reviewing and processing on any workstation. With the reviewing software Osiris ${ }^{28}$ available as the standard HUG-PACS display environment, the registered data sets can then be visualized in side-byside or screen-mesh fusion modes, and regions of interest or other primitives can be drawn on one data set and projected onto the others for calculations or localization.

Besides the Papyrus files, all the objects created for display on the MM workstations can also be saved as images or as $3 \mathrm{D}$ objects under the OpenInventor ${ }^{29}$ or virtual reality modeling language VRML ${ }^{30}$ standards. These objects can then be transferred and used for illustration or navigation through other commerical or public domain multiplatform software.

\section{Hybrid Registration of Multisensor Data Sets}

IMIPS (Fig 2) was developed in Geneva primarily to allow for the retrieval, registration, and $3 D$ to $5 \mathrm{D}$ fusion of 

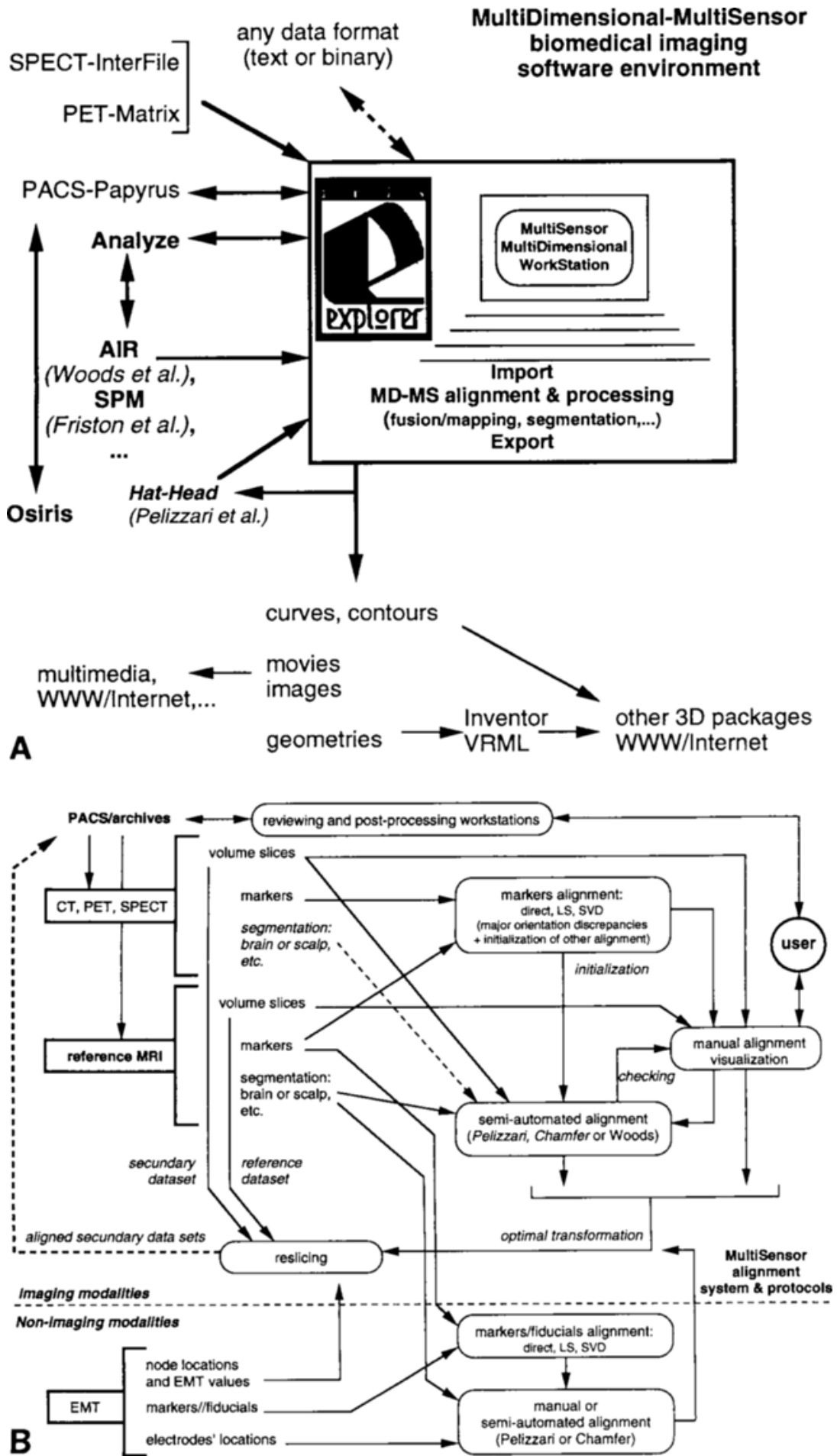

Fig 2. (A) Multidimensionalmultisensor environment and software for the study of brain anatomy, metabolism and function. (B) The whole system and protocols are all grouped within IMIPS.

morphological data, such as bone and soft tissue anatomy extracted from CT, brain anatomy extracted from MRI, angiography data from MR (MRA) or CT (CTA), and functional data modalities, such as PET, SPECT, or EEG/ MEG-based EMT.

As stated in the introduction, there is no general solution to registering two data sets, and several established techniques of complementary types were implemented in IMIPS so that the investigators would be able to solve most registration problems by optimally combining these techniques with their data.

Whenever possible, a semiprospective protocol is used 
that initializes the registration of the more traditional imaging modalities (MR, CT, PET, and SPECT) by a geometric transformation, making use of commercial external MM markers (IZI, Baltimore, MD). The markers are used as they come for CT and MR (they behave like skin), but require a small dose of radiotracer $(\approx 0.2 \mathrm{~mL}$ of $a \approx 30$ $\mu \mathrm{Ci} / \mathrm{mL}$ solution) for SPECT and PET acquisitions. They are placed at the nasion and preauricular cavities for every acquisition. These locations have been extensively used as references in many $\mathrm{MM}$ projects. ${ }^{31}$ as they are easy to identify and do not move significantly during or between examinations. Currently, the authors also use a fourth "orientation" marker on the right temporal cavity for solving the possible orientation discrepancies (eg, resulting from different equipment or protocols) between the various modalities being considered.

The markers are clearly visible in every data set, and their location can easily be extracted by interactive pointing on the reconstructed images. From these locations, the geometric transformation from one set of markers to another one can be calculated either directly (with only three markers to consider) or through techniques that can be extended to use more landmarks. ${ }^{13,14}$

From the "gross" initial marker-based alignment step, the alignment transformation can be refined either manually or semiautomatically. The manual alignment techniques, through interactive $3 \mathrm{D}$ surface manipulation or direct transformation input (ie, 3D translations and rotations in the markers' or initial volume's referential), are still the standard against which all other techniques are compared. ${ }^{12,32}$ Even with the help of the marker-based initialization step and reasonable interaction speed, they can be tedious to use. For this reason, and also to rely less on the possibly exclusive expertise of a few users for the bulk of routine cases, other more automated means of objectively-. and iteratively-refining the alignment between modalities have also been implemented in IMIPS including 3D surface approaches, ${ }^{16}$ 3D (Chamfer) distance maps, ${ }^{17: 18}$ or data similarity ${ }^{2}$ approaches. These individual techniques, which often imply a preliminary segmentation step, are all virtually integrated in a hybrid global system (similar to Bidaut et $\mathrm{a}^{33}$ ), where they are used to solve the specific alignment steps on which they perform best, to complement or support the other techniques. Figure $2 \mathrm{~B}$ explains better how the various components of IMIPS interact to register specific MS pairs.

Whenever the original data sets contain similar enough information (cg, anatomic clues), the quality of the alignment can be assessed at anytime by visualizing overlapping slices or 3D surfaces of similar structures (Fig 3).

Registration of EMT to MR. The standard T1-weighted 3D MRI acquisition used as spatial reference is performed on the patient at some time close to the EEG. Before the EEG recording, all electrode locations are digitized with a 3D digitizer, such as a Polhemus magnetic system (Polhemus Laboratories, Inc, Colchester, VT) or a passive robotic arm (Fig 4A). To simplify the alignment step, more anatomic or fiducial landmarks (such as the markers intro-
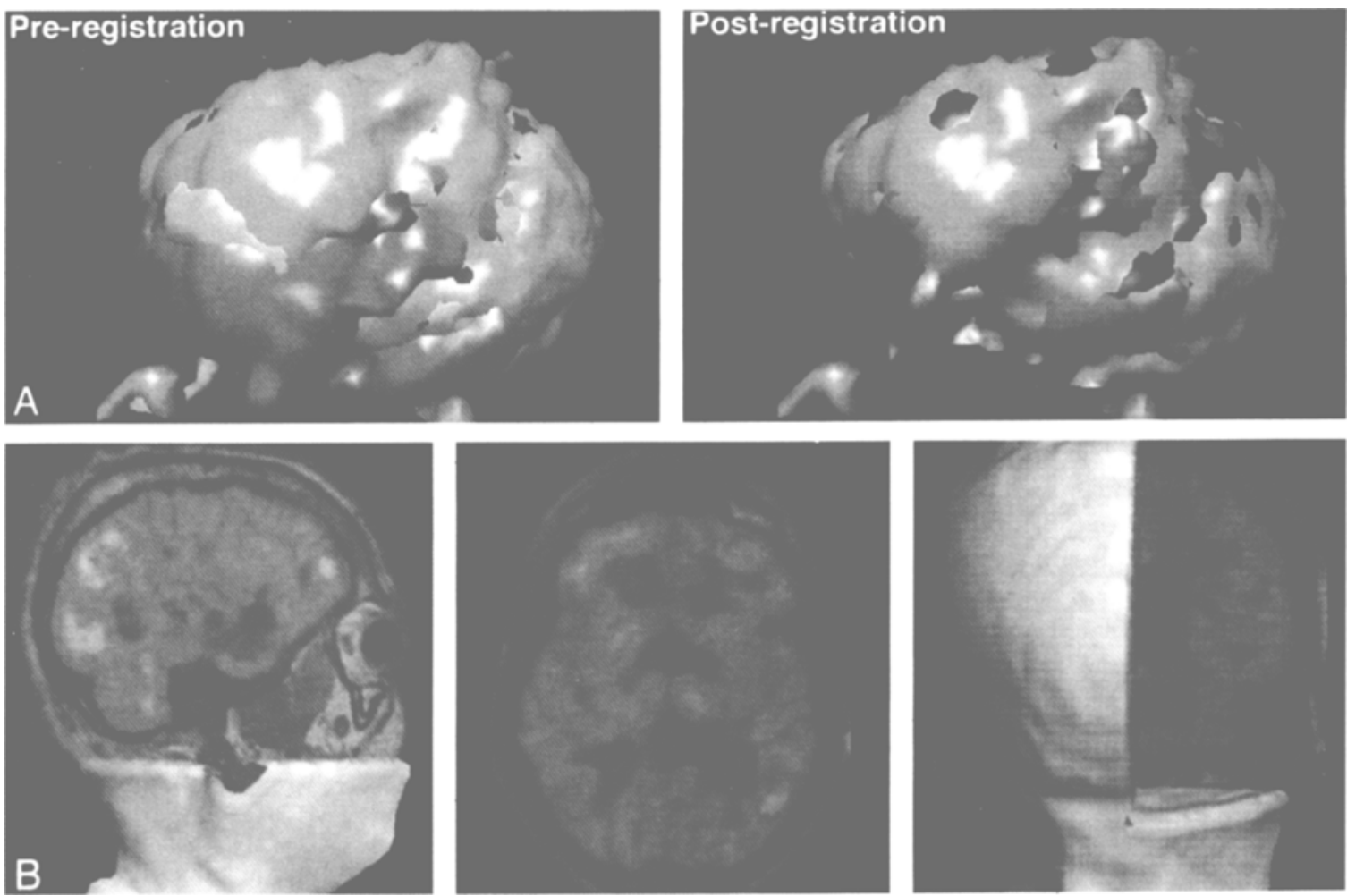

Fig 3. Verification of multimodality alignment: surface based (A, brain surfaces from four SPECT data sets) and slice based (B, MR and SPECT data sets). 

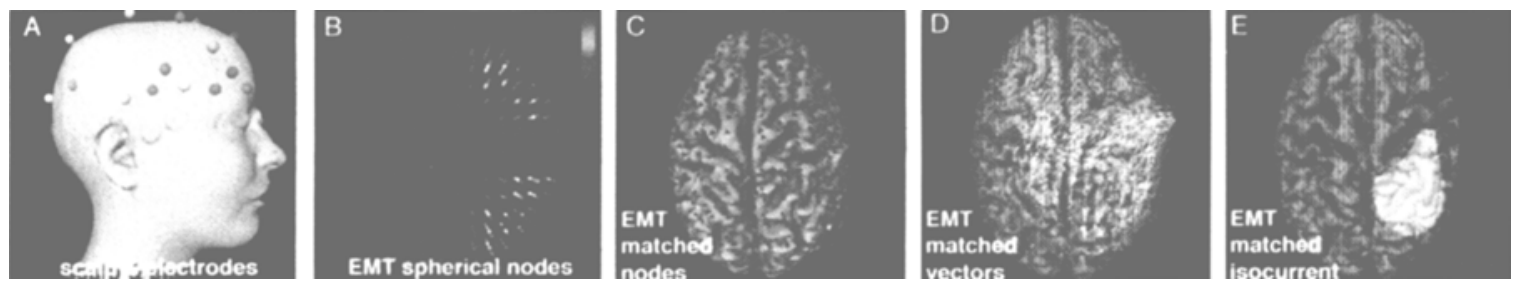

Fig 4. (A) MR scalp and EEG electrodes. (B) EMT spherical reconstruction nodes. (C) EMT nodes matched to the actual brain anatomy. (D) Matched EMT vector field. (E) Matched EMT maximum current envelope.

duced previously), which can easily be identified on the reference MRI data set, can also be digitized at this stage. It is worth mentioning that the quality of the EMT-MR alignment is dependent on the quality of this measurement.

As the reconstructed EMT maps in their ideal spherical virtual space ${ }^{25}$ (Fig 4B) do not contain enough information about the underlying anatomy, the fiducials and electrodes' locations on the scalp recorded before the EEG (Fig 4A) are the only information allowing one to register the EMT maps to the actual MR anatomy. Because of the presently low number of electrodes $(\approx 27$ in current long-term monitoring settings), the alignment is performed either manually from the initial transformation provided by the markers' locations (ie, by $3 \mathrm{D}$ rotations and translations) or with the help of an iterative search (similar to Pelizzari et $a^{16}$ or Mangin et $\mathrm{al}^{18}$ ), which minimizes the 3D distance between the electrodes' "cloud" and the scalp segmented from the reference MR data set.

In addition to the EMT-MR alignment, the electrodes' locations (Fig 4A) are also used to resample spatially, in the real 3D space where they were initially located, the EMT data reconstructed inside their perfect virtual sphere. To do so, we use the initial transformations that map every electrode onto the surface of the virtual EMT head sphere before the EMT reconstruction. ${ }^{25}$ These transformations are inverted so that the EMT reconstruction nodes, which are uniformly sampled throughout the virtual reconstruction sphere (Fig 4B), now have actual 3D space coordinates within the volume enclosed inside the scalp surface as defined by the electrodes' true locations (Fig 4C and 4D).

\section{Reslicing of Multisensor Data Sets}

Once found, the optimal interset geometric transformations can be applied to the initial data sets for reslicing them individually to match the reference one spatially (eg, MRI). As the modalities of interest carry different types of information, this reslicing can be performed with different modes likely to best preserve this individuality. Using all possible a priori knowledge about the information to be best preserved during the reslicing (eg, hyposignal or hypersignal), this operation can be done by either nearest, trilinear interpolation or standard ranking (minimum, median, or maximum) filters ${ }^{34}$ on the trilinear kernel. To improve the appearance of the resliced data sets, anisotropic data may also be interpolated before the reslicing itself by linear interpolation or ranking filtering.

Reslicing of the EMT data. After registration of the fiducials' or EEG electrodes' locations between the MR and EMT data sets (Fig 4A), the four-dimensional (4D) EM data (true spatial 3D and time $=4 \mathrm{D}$ ) are transformed through the optimal geometric alignment transformation into the MRI referential. Because of the low resolution of the EMT reconstruction, the reslicing can take several forms, depending on the information to be emphasized: nearest node value, trilinear interpolation, or ranking (minimum, median, or maximum) filters between closest adjacent nodes. Because of the low resolution and coarse or nonuniform sampling of the EMT nodes in the real 3D space, every reslicing mode comes with a "capture sphere" (CS) option that controls the contribution of every EMT node to the resliced voxels (elementary elements of volume). The CS makes sure that the EMT nodes are considered within their actual spatial spreading range, which is a function of both the physical measurement geometry (eg, EEG) and the EMT reconstruction technique. Setting the CS radius at any individual EMT node to match the resolution of the virtual EMT reconstruction at this location ensures that every EMT node contributing to a specific spatial reference voxel will be properly considered in the EMT reslicing calculations.

\section{Multisensor Visualization}

Several techniques can be used to visualize or manipulate the 3D to 5D MS data sets resulting from the IMIPS approaches. ${ }^{35,36}$ Most figures presented in this report demonstrate the capacity of the tools that have been implemented in IMIPS to take care of visualizing in 2D or 3D any combination of information extracted from a 5D MS volume.

Directly from the MS space, slices from every sensor can be presented either side by side or in "colorwash" mode, the latter being a superposition and transparency fusion of individually colored slices (see Figs 3, 7 and 8). Because of the CS concept, the resliced EMT images (see Fig 8A) actually give clues as to how coarse the EMT node sampling is in a specific area or how distant a resliced voxel is from any initial EMT node. The resliced EMT data show nonoverlapping circular shapes in the areas of low EMT spatial resolution and in locations distant enough from actual EMT nodes (eg, edges of the brain), while giving apparently more densely packed voxels in other areas (eg, inner brain). In the images of Fig $8 \mathrm{~A}$, where the information to preserve was an EMT hypersignal, the CS radius was set to the minimum distance between distinct EMT nodes so that the already low EMT resolution could be kept at its highest.

The brain or other structures can also be segmented in IMIPS from the 3D reference MRI data set by manual or automated morphotopological segmentation (similar to the 
one described in Mangin et $\mathrm{a}^{18}$ ). The latter, which combines thresholding and mathematical morphology concepts, is robust enough for segmenting the whole brain and seldom requires manual edition of its results. The masks and surfaces produced at this step can later be used in subsequent processing or visualization.

All 3D to 4D surfaces extracted from the MS space can be rendered and manipulated in $3 \mathrm{D}$ with OpenInventor ${ }^{29}$ or VRML ${ }^{36}$ compatible multiplatform viewers. They can also be made transparent to better show their relationship with inner structures or slices from the MS volume. For example, see Figs 4E, 5, 7B and 8B, which show isocurrent surfaces extracted from the realigned EMT data. Depending on the information of interest in the EMT, and also if a complete time sequence is being investigated, the threshold used to caiculate the isocurrent surface can either be absolute (on a global $4 \mathrm{D}$ sequence) or relative (on the single $3 \mathrm{D}$ time frame under scrutiny). To provide in a 3D mode some information of a higher dimension, the investigators can also show a color-coded $3 \mathrm{D}$ to $4 \mathrm{D}$ projection of any sensor (eg, metabolism from PET-SPECT or reconstructed EMT current densities through time) onto the brain structures extracted from the morphological MR data set (see Figs 5 and 9).

\section{RESULTS}

The techniques and protocols implemented in IMIPS now allow one to retrieve, register, and integrate on a routine basis many medical data of a definite complementary nature. Once they belong to the same 5D space, data from any multisensor volume can easily be reviewed, visualized, compared, and used in further processing, which makes the IMIPS approach totally compatible with either clinical or research applications. The modular design of IMIPS allows easy implementation of any new technique or component within the global system at hand, as soon as they become available or necessary.

\section{Validation of EMT-MR Registration}

EMT is the least imaging modality of the ones currently in use in IMIPS and is a new entry in the MS field. For these reasons, the correlation between the 4D EMT maps and the brain areas known to respond to specific stimuli in normal subjects has been verified by using evoked response potential (ERP) data.

The ERP experiments shown in Figure 5 are better described in Pascual-Marqui et al. ${ }^{25}$ The stimulus retained for illustration is a visual one: right hemiretina checkerboard (rHRCB). Specifically, for the rHRCB test, there are peaks in the electrical measurements at 100 and 136 milliseconds poststimulus. These instants, which exhibit a spatial inversion in the localization of the maximum EM activity, are the ones that have been retained in Fig 5 to demonstrate both the timing and localization capabilities of the EMT within the more global multisensor proto$\mathrm{col}$, as well as various ways of representing these data in relation to the underlying anatomy. The slight nonpredominant frontal activity seen in the EMT is actually not significant for the experiment and is principally due to an EMT artifact caused by the uneven sampling (targeted at occipital/visual cortex regions) of the electrodes retained for the EMT calculations.

\section{Clinical Examples}

In addition to oncology, ${ }^{37}$ where $\mathrm{MM}$ applications have already been largely advocated, ${ }^{1,2}$ many drug-resistant epileptic patients have already been investigated. The authors' clinical epilepsy assessment protocol (Fig 6) is primarily intended for presurgical evaluation of the patients. It currently includes 3D MRI (standard T1-weighted, $1 \mathrm{~mm}^{3}$ voxels), interictal FDGPET, ${ }^{22,23}$ interictal SPECT (initially with HMPAO and now with ECD, which is much more stable through time), ${ }^{23,24}$ long-term EEG monitoring with video recording, and ictal ECDSPECT (injection $<10$ minutes after the beginning of a crisis detected by EEG). Other MR sequences or functional acquisitions (fMRI, post-ictal SPECT, etc) may also be included whenever needed or available.

Two typical cases are described briefly in this report to illustrate the MS investigation the patients undergo and the clinical benefits it can provide.

Patient 1 has a lesion (cavernoma) that can be easily seen on the MR data set (Fig 7). PET and SPECT, respectively, show focal hypometabolism and hypoperfusion in the area of the lesion (Fig 7A). EMT maps at various moments during the ictus (Fig 7B), and particularly at the onset, always show an elevated EM activity in the area of the lesion. Although the PET and SPECT findings (which are not epilepsy related) do not permit any conclusion to be reached regarding the epilepsy source, the EMT findings point to the functionally involved lesion as the most probable source of epileptic activity. 
The outcome of this investigation will be a resection of the lesion, with the help of the MR spatial reference to localize it better.

Patient 2's MR does not show any visible lesion or tissue damage. Ictal SPECT and EMT both show an area of increased perfusion and EM activity in the frontal cortex (Fig 8A). The spatial gap between the two sites seen in Fig $8 \mathrm{~B}$ is not truly significant and is further explained in the Discussion.

For this patient too, other modes of MS visualization are also shown (Fig 8) with the projection of ictal SPECT and EMT data on the outer and inner surface of the cortex extracted from the MR data set. These modes visualize both sides of the cortex, and allow for better assessment of the relationship between the right frontal SPECT hyperperfusion and the maximum EM activity during the ictus regarding the surrounding neurological anatomy (eg, sulci). Even retrospectively, there are no visible lesions on the morphological MR data set at the location of the functional abnormality. As some EM activity (while of lesser amplitude) is also measured at other locations during the ictus, depth electrodes will be implanted with the help of the multisensor data. Their electrical measurements will then be used to probe for other possible locations for the epileptic trigger(s) or focus (Fig 9).

\section{DISCUSSION}

$M M$ registration. The $\mathrm{MM}$ alignment and reslicing aspects of the IMIPS system described in this report have been designed to minimize changes to the current routine acquisition protocols while allowing the use of the data in the most complex MS protocols. As such, minor adaptations were necessary to allow for the proper use of a few markers to resolve orientation differences between the different data sets and provide a good initial interset geometric transformation for subsequent alignment steps. This basic setting should be kept as long as the data sets do not carry reliable orientation information or are not similar enough across examinations. For example, the use of much different (and multivendor) tomography equipment with their own data orientation (ie, left/right, head/ foot) does not always allow for the easy absolute orientation of the initial data sets. In the near future, it is hoped, a wider use of medical file standards $^{27}$ should efficiently solve the problems of spatial compatibility by propagating appropriate reference and orientation data structure along with every data set. Also on the bright side, the lack of significant distortion from the tomographic imaging modalities (as measured by standard quality control procedures) obviated the need for a specific geometry correction component, such as coordinate scaling or warping. ${ }^{38}$

Working with only a few markers and also with low resolution (and often anisotropic) imaging devices, the geometric transformation extracted from the markers alone does not allow for perfect alignment of the data sets. Nevertheless, the initial result takes care of the orientation differences and is near the ideal solution. This proximity then helps itera-

Fig 5. Visual ERP (right hemiretina checkerboard) EMT at two selected instants. EEG potentials recorded during a rHRCB ERP exhibit two peaks at 100 and 136 milliseconds (see EEG curves). The EMT reconstructions at these two moments show the characteristic inversion of the maximum EM activity from the right side (100 milliseconds) to the left side (136 milliseconds) through the display of the maximum current envelope. The other display modes allow for better assessment of the link between the EM activity and the undertying anatomy (outer and inner cortex). Although only two characteristic time frames have been selected for this figure, each single time frame (every millisecond for an ERP) can be projected in the same way to follow dynamically the evolution of the EM signal through time and in relation with the anatomy.

Fig 7. Patient 1: (A) Multimodality slices showing PET hypometabolism and SPECT hypoperfusion at the location of the lesion seen on the MR slice. (B) Four different moments following the onset of an ictus; the EMT current during the discharge always stays at an elevated value (as shown by the EMT maximum current envelope) in the area of the lesion (at the accuracy of EMT).

Fig 8. Patient 2: (A) Multimodality slices; the slices exhibit an ictal SPECT hyperperfusion (middle column) and an ictal EMT hypercurrent (right column). The appearance of the EMT slices is further described in the article. (B) Comparison of the SPECT and EMT data, and the locations of maximum EMT current and maximum SPECT perfusion.

Fig 9. Patient 2: Projection of functional data (EMT [top row] and ECD-SPECT [lower row]) on the patient's brain segmented from the MR data set. The left column shows the projection of the functional data on the outer surface of the cortex. The right column shows the inner surface of the cortex $1 \approx$ outer surface of the white matter $)$, which is more closely related to the sulci and shows a different side of the relation between functional data and underlying anatomy. For this patient, the interest of the mapping is to show the relation between the anatomy and maximum EM activity, and metabolic perfusion seen in the right frontal cortex during the ictus. 

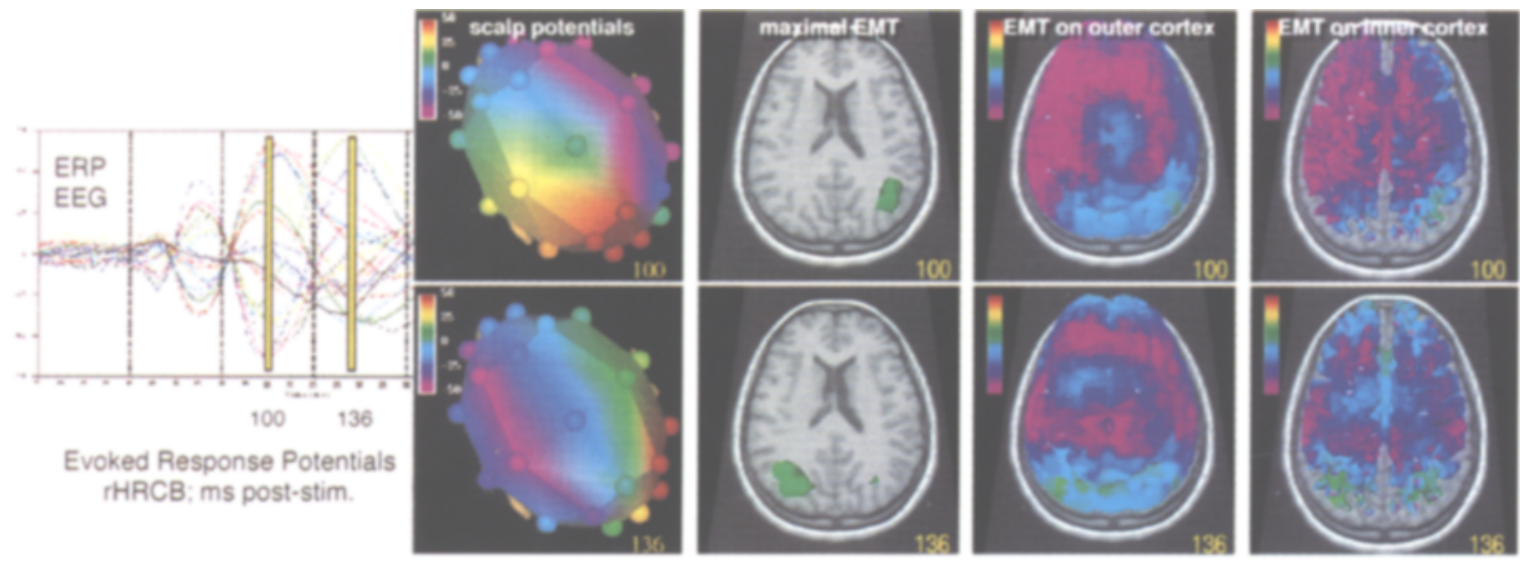

Figure 5
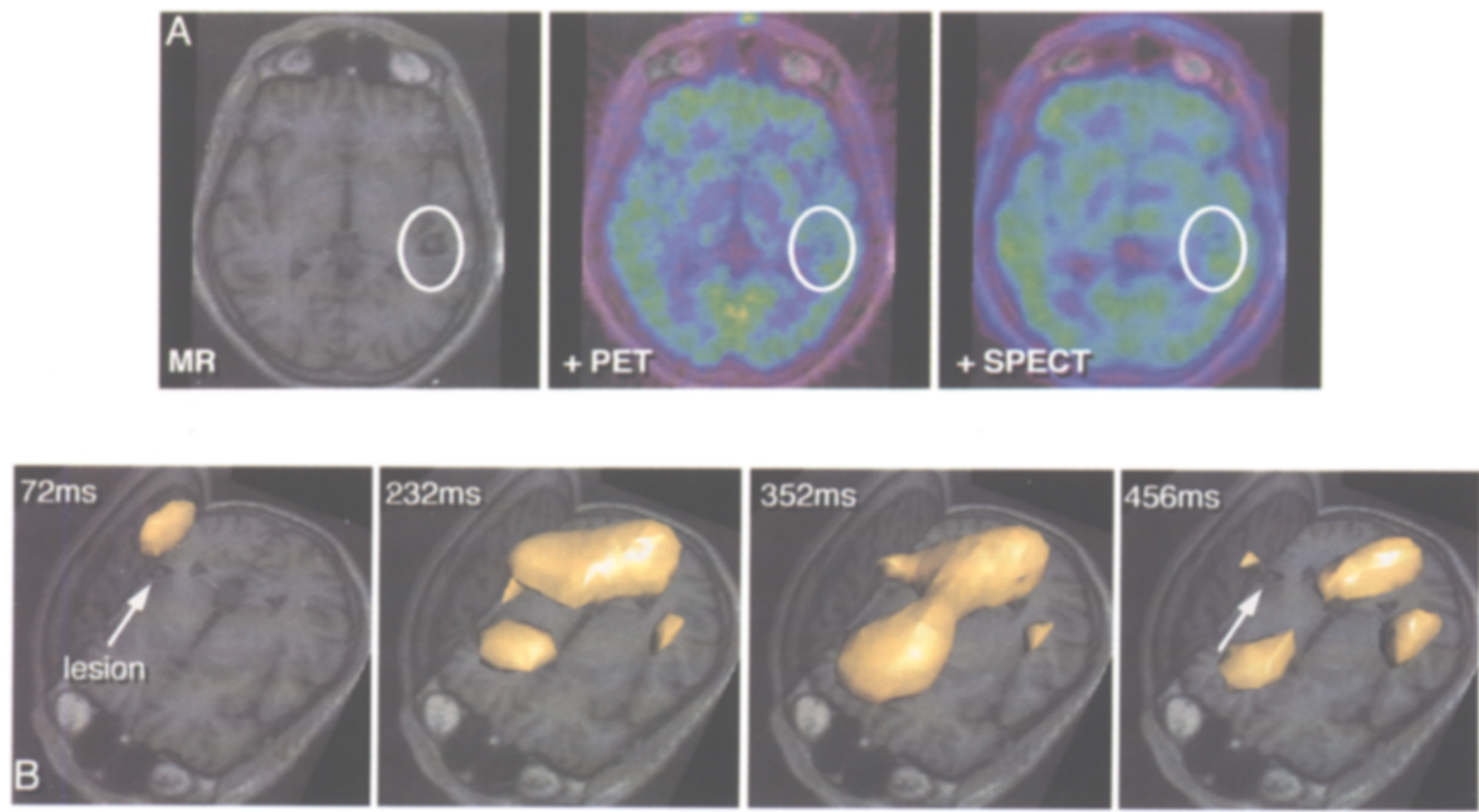

Figure 7
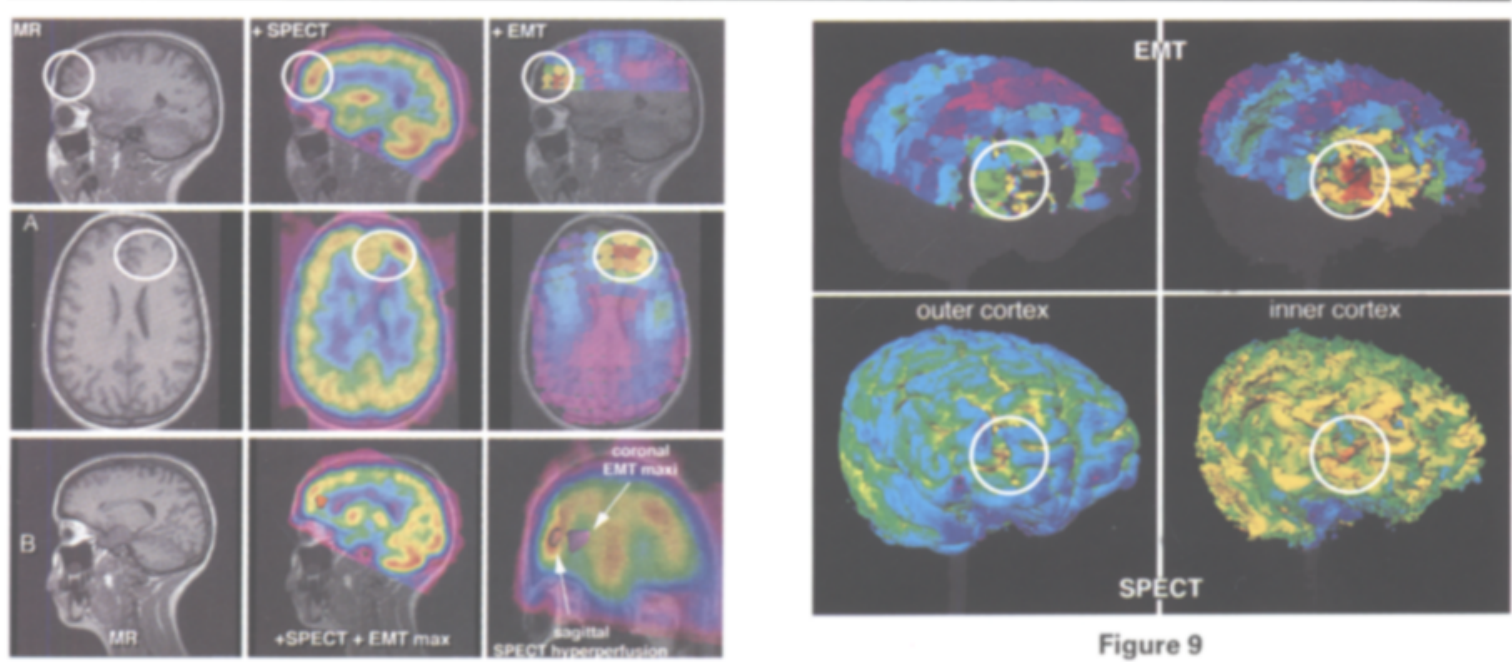

Figure 9 


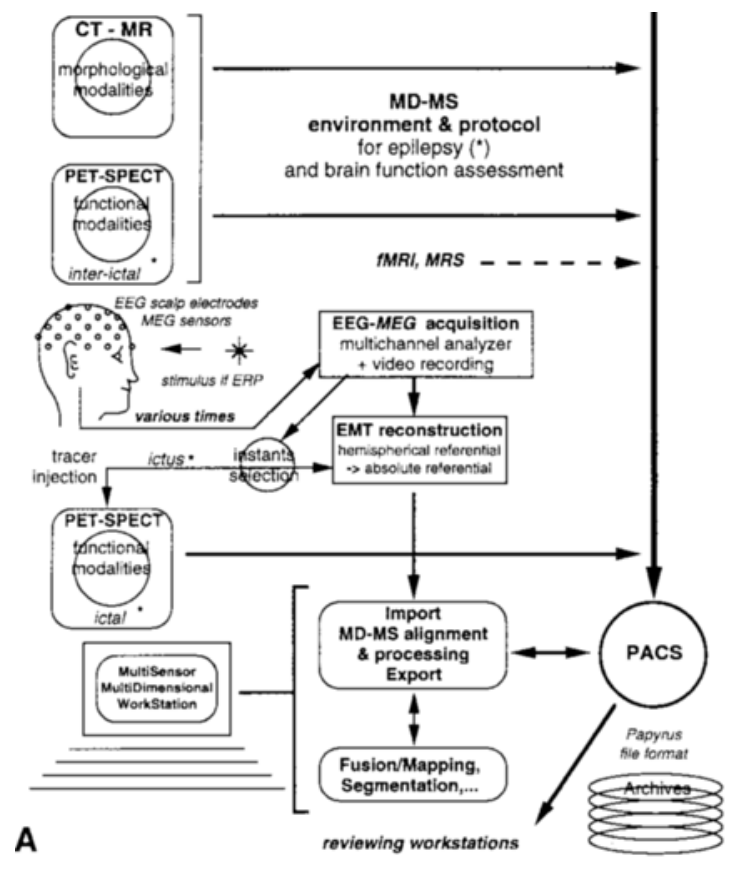

tive miethods to avoid local minimas in which they could be trapped-far from the actual solution.

The IMIPS multicomponent alignment approach was built on published and recognized developments, the accuracy of which is strongly dependent on the resolutions and types of the modality pairs under scrutiny. By using a preliminary marker step, a final possible manual interaction, a best-choice method, and state-of-theart acquisition devices (which were not always available at the time of the initial developments), registration solutions should obtain an accuracy similar or better than the original published values. ${ }^{12,32}$ Still, and as actually mentioned in the original publications, ${ }^{12,32}$ alignment results occasionally require visual checking and final manual correction, whenever enough similar clues are visible on both data sets (which is the case with the sequences and tracers we are currently using). This "poor performance" is generally due to spatial discrepancies between the incoming data sets (eg, too much anisotropy) or to too many differences in the interset features used by the alignment methods (eg, incomplete brain coverage). When not specifically linked to intrinsic limitations, such as tracer choice in PET or SPECT, these

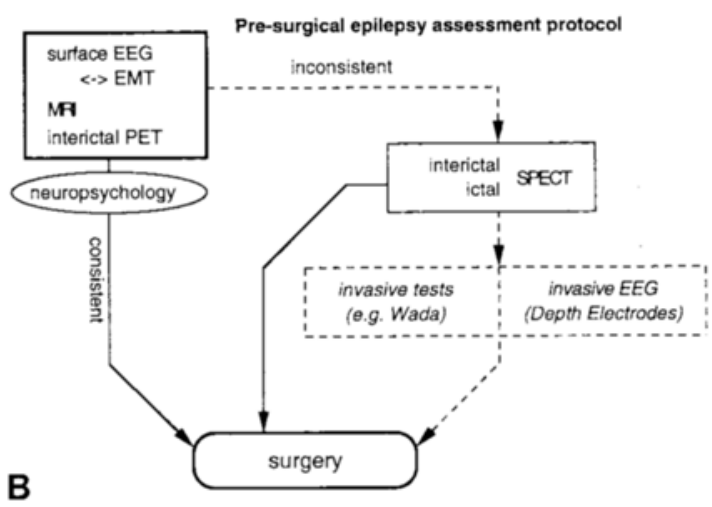

Fig 6. Epilepsy protocol environment (A) and decision tree (B). The asterisks in Fig $6 \mathrm{~A}$ mark the steps required for the presurgical assessment of epilepsy.

differences can sometimes be anticipated and corrected at the initial acquisition/reconstruction time by clearly stating before the individual examinations which ones are planned to be used in a MS protocol.

EMT-MR registration. The EMT reconstructed data are of low resolution; in its present setting, LORETA can resolve two point sources if separated by more than $25 \mathrm{~mm}$. With this intrinsic limitation, the simple nasion and preauricular fiducials' alignment of an EMT map with the MR spatial reference generally gives adequate results. Even so, manual interaction can still be necessary to correct for possible problems, such as patient motion during digitizing of the electrodes' locations. The limited number of electrodes in the current routine EEG recording protocol $(\approx 27)$ does not allow much better results to be reached when iteratively minimizing, by techniques, such as those used by Pelizzari et al ${ }^{16}$ or Mangin et al, ${ }^{18}$ the distance between the electrodes' locations and the relatively smooth $3 \mathrm{D}$ scalp surface extracted from the MR data set. If, as it is planned, the number of electrodes is increased from 27 to 64 or even 128 , the more automated iterative alignment techniques might give better results, compatible with a higher-resolution EMT reconstruction. 
Reslicing. The ranking filters reslicing interpolation modes are an attempt to make interpolation a little smarter without going to the expense of full shape-based interpolation, ${ }^{39}$ which would require preliminary segmentation of the data sets. Although the latter interpolation technique certainly produces better results, at least near the edges of the structures of interest, segmentation of the initial shapes is not always possible based on only single modality data sets. Besides, one should always remember that interpolation does not objectively improve the initial content of the data. Its major goal is attempting to re-create, under reasonable assumptions, what is missing or has been lost at previous steps.

Visualization. By going further in the segmentation of the brain by windowing MR signal values or going to more statistical approaches, ${ }^{40,41}$ the values of the nearby EM or metabolic activity can be mapped onto the outer and inner surfaces of the cortex (Figs 5 and 9) or even more specific structures within the brain. As it stands, the ability to peel away the cortex (from outer cortex to outer white matter) and project on these anatomic structures data from other sensors is expected to improve the understanding of brain function. Sulci segmentation and identification ${ }^{42}$ will eventually further this approach.

Validation and clinical examples. Investigations, including depth electrodes EEG, are still being conducted to assess the correlation between structure and function (by PET, SPECT, and other EEG methods as well as by EMT) better for pathological conditions, such as epilepsy.

Although some patients exhibit morphological lesions, which are easily identified on the routine MRI and likely related to their epilepsy, others do not present any morphological anomaly. For these latter patients, the information brought in by the functional modalities is absolutely necessary to further the investigation. Even if PET alone does not show any local hypometabolism, interictal and ictal SPECT can still be compared, either qualitatively (eg, by normalizing the ECD uptake values to the cerebella hemispheres in both data sets) or semiquantitatively (eg, by histogram-based inter- set normalization or by kinetic modeling approaches). In the two clinical examples presented in this report, it is interesting to notice the slightly different type of complementary information that comes out of the MS approach for the two types of patients. In cases like patient 2, preliminary MS results are not conclusive enough, and further investigations need to be performed (eg, with the help of implanted depth electrodes or more targeted long-term scalp EEG monitoring). Also, as qualitative measurements are not always enough to assess tissue function properly, ${ }^{43,44}$ an effort is under way to integrate more quantitative procedures and data (pharmacokinetic modeling approaches for PET and SPECT, statistics, etc) within the global MS space currently dealt with by IMIPS.

In Fig $8 \mathrm{~B}$, the spatial gap between the two sites of maximal EMT current and SPECT perfusion is well within the limits of the EMT resolution accuracy. Also because of noticeably different intrinsic principles between the two sensors (particularly their distinct timing capabilities), the sites can reasonably be considered as spatially related. Moreover, the dynamic information provided by the "motion" of the EM maximum activity during the ictus could actually be used to highlight specific areas of the brain with "slower" criteria than instant EM activity. The new parametric areas might then be compared with MR morphology or function, and with SPECT or PET (quantified) metabolism.

Intra-patient versus inter-patient. For the time being, the IMIPS protocols and system presented in this report have been designed primarily to accommodate analyses performed on single individuals. Although IMIPS performs the task well, there is a definite need to expand it further to interindividual processing and analysis for studying neurological function and metabolism in a more global population. Such an expansion is occurring in two separate directions to accommodate the diversity and specificity of the investigations. The first direction is to use an atlas-based approach, such as statistical parametric mapping, ${ }^{45}$ whereas the second approach tries to address the interindividual anatomic disparities better by using approaches, such as described in Mangin et $\mathrm{al},{ }^{42}$ as a frontend to more complex statistics. 
EMT related issues. Although LORETA is currently being used in Geneva for EMT calculations, other techniques (such as commercially available time-varying dipole modeling ${ }^{46}$ ) exist to reconstruct EM data from EEG or MEG measurements. Although these techniques all try to address similar issues in attempting to find "the" best solution to the inverse problem of localizing the physiological generators from the physical measurements, they rely on fundamentally different principles. As IMIPS is not limited to a single EMT method, a direct comparison of these methods is beyond the scope of this article.

In subsequent developments, refinements should and will be made in some aspects of the EMT reconstruction, at least to further its validation in parallel with the other modalities. These refinements are actually similar to the ones already occurring in the other functional and metabolic imaging fields where CT and MR data are expected to improve the reconstruction and processing of PET, SPECT, and fMRI data.

The "capture sphere" concept developed in IMIPS for the reslicing of EMT data is evolving toward a more realistic shape that should better consider the actual non-uniform spatial sampling and spreading of the EMT nodes in all three dimensions. The EMT coarse sampling is both due to the nonuniform electrodes' spacing and to the EMT reconstruction assumptions. As currently done for ERP experiments, the electrodes' locations can be tuned to cover better specific locations, such as the temporal lobes or other areas under scrutiny. From this locally improved spatial sampling at the physical measurement level, the EMT calculations can then also provide a better spatial sampling at the reconstruction level in the selected areas. As the EMT investigation for epilepsy is currently being performed in parallel with the routine long-term neurological investigation, the number of electrodes is relatively low and does not evenly cover the whole brain. In such a setting, LORETA, which can make use of any additional information, such as depth electrode measurements, still reconstructs, although with low resolution, all sources within the volume and can discriminate between individual maximums if they are at least separated by approxi- mately $25 \mathrm{~mm}$. Modifying the number of electrodes or tuning the electrodes' spatial sampling to more specific areas would modify this discrimination distance.

\section{CONCLUSION}

The open and modular MS facility (IMIPS) has been developed and implemented in Geneva to take care of all needs for both clinic- and research-oriented MS imaging and data processing.

Besides efficiently addressing the clinically compatible registration issue for various sensor pairs, IMIPS also includes some powerful visualization concepts and tools to present the information from a 5D MS space in a way understandable by both scientists and nontechnically oriented physicians.

The authors have shown the feasibility and potential interest of including - through means easily implemented in the clinical routineEEG(-MEG) EMT in an already complex and rich (although more traditional) MM space that includes MR-CT anatomy and PET-SPECT metabolism. Besides FDG-PET and SPECT, which are now performed on a routine basis, 3D echo-planar fMRI and MRS should also be soon available in Geneva, along with better access to more marked PET molecules produced in a new radiochemistry laboratory. In its present shape, but even more so by including MEG, IMIPS can already be extensively used for studying normal and pathological brain metabolism and function, for example, through ERP's and other functional modalities (PET or fMRI).

In Geneva, the authors decided to target initially only a limited number of protocols with an expected added value from MS approaches. The MS grading and therapy planning of tumors and the assessment of pharmacoresistant epilepsy are such protocols. In addition to providing an improved opportunity to gain a better knowledge of the underlying in vivo metabolism and (dys)function of the tissues, the anatomically related multisensor space should also allow for better planning and monitoring of any subsequent therapy. Further protocols will be introduced as the authors learn better from the ones on which they initially focused. 
When considering all the functional sensors now at hand, the total richness of the resulting combined MS space has not yet been fully appreciated at the moment. The newly envisioned MS potential, primarily based on multidisciplinary approaches, should never hide the complexity of putting together data (and people) from many scientific and technical fields, even for rather targeted research protocols. Only by properly assessing the means and educating the potential users will prevention of the disappointments that might come from less reasonable expectations be possible.

\section{ACKNOWLEDGMENT}

The authors thank Prof D. Lehmann for providing the EEG-ERP recordings initially used for EMT-MR validation.

\section{REFERENCES}

1. Wahl RL, Quint LE, Cieslak RD, et al: Anatometabolic tumor imaging: Fusion of FDG-PET with CT or MRI to localize foci of increased activity. J Nucl Med 34:11901197, 1993

2. Pirotte B, Goldman S, Bidaut LM, et al: Use of positron emission tomography (PET) in stereotactic conditions for brain biopsy. Acta Neurochir 134:79-82, 1995

3. Pomeranz SJ, Pretorius HT, Ramsingh PS: Bone scintigraphy and multimodality imaging in bone meoplasia: Strategies for imaging in the new health care climate. Semin Nucl Med 24:188-207, 1994

4. Freitas JE, Freitas AE: Thyroid and parathyroid imaging. Semin Nucl Med 24:234-245, 1994

5. Alexander C, Villena-Heinsen CE, Backens M, et al: Image fusion of MRI and immunoscintigraphy with MAb170 in ovarian tumors. Nuklearmedizin 34:173-178, 1995

6. Fox PT, Woldorff MG: Integrating human brain maps. Curr Opin Neurobiol 4:151-156, 1994

7. Tamraz J: Neuroradiologic investigation of the visual system using magnetic resonance imaging. J Clin Neurophys 11:500-518, 1994

8. Brunetti A, Soricelli A, Rotondo A, et al: Correlative morphologic and functional imaging for diagnosis, staging and follow up in AIDS: An overview. Q J Nucl Med 39:201-211, 1995

9. Elgazzar AH, Abdel-Dayem HM, Clark JD, et al: Multimodality imaging of osteomyelitis. Eur J Nucl Med 22:1043-1063, 1995

10. Ratib O, Didier D, Chatelain P, et al: Standard views in cardiac multimodality tomographic imaging. Am J Card Imaging 9:67-76, 1995

11. Ferris JV, Marsh JW, Little AF: Presurgical evaluation of the liver transplant candidate. Radiol Clin North Am 33:497-520, 1995

12. Van den Elsen P, Pol E-JD, Viergever MA: Medical image matching: A review with classification. IEEE Eng Med Biol 12:26-38, 1993

13. Arun KS, Huang TS, Blostein SD: Least square fitting of two 3D point sets. IEEE Trans Pattern Mach Intell 9:698-700, 1987

14. Umeyama S: Least square estimation of transformation parameters between two point patterns. IEEE Trans Pattern Mach Intell 13:376-380, 1991

15. Dhawan AP, Arata LK, Levy AV, et al: Iterative principal axes registration method for analysis of MR-PET brain images. IEEE Trans Biomed Eng 42:1079-1087, 1995

16. Pelizzari CA, Chen GTY, Spelbring DR, et al:
Accurate three-dimensional registration of CT, PET, and/or MR images of the brain. J Comput Assist Tomogr 13:20-26, 1989

17. van Herk M, Kooy HM: Automatic three-dimensional correlation of CT-CT, CT-MRI, and CT-SPECT using chamfer matching. Med Phys 21:1163-1178, 1994

18. Mangin J-F, Frouin V, Bloch I, et al: Fast nonsupervised 3D registration of PET and MR images of the brain. $J$ Cereb Blood Flow Metab 14:749-762, 1994

19. Woods R, Mazziotta JC, Cherry S: MRI-PET registration with automated algorithm. J Comput Assist Tomogr 17:536-546, 1993

20. Meyer CR, Leichtman GS, Brunberg JA, et al: Simultaneous usage of homologous points, lines and planes for optimal 3D linear registration of multimodality imaging data. IEEE Trans Med Imaging 14:1-11, 1995

21. Alpert NM, Berdichevsky D, Levin Z, et al: Improved methods for image registration. Neuroimage 3:10-18, 1996

22. Gaillard WD, White S, Malow B, et al: FDG-PET in children and adolescents with partial seizures: Role in epilepsy surgery evaluation. Epilepsy Res 20:77-84, 1995

23. Nagata $T$, Tanaka F, Yonekura $Y$, et al: Limited value of interictal brainperfusion SPECT for detection of epileptic foci: High resolution SPECT studies in comparison with FDG-PET. Ann Nucl Med 9:59-63, 1995

24. Matsuda $\mathrm{H}$, Yagishita A, Tsuji S, et al: A quantitative approach to technetium-99m ethyl cysteinate dimer: A comparison with technetium-99m hexamethylpropylene amine oxime. Eur J Nucl Med 22:633-637, 1995

25. Pascual-Marqui RD, Michel CM, Lehmann D: Low resolution electromagnetic tomography: a new method for localizing electrical activity in the brain. Int J Psychophysiology 18:49-65, 1994

26. Ratib O, Ligier $Y$, Scherrer J-R: Digital image management in medicine. Comput Med Imaging Graph 18:73-84, 1994

27. Bigwood WD, Horii S: Introduction to the ACRNEMA DICOM standard. Radiographics 12:345-355, 1992

28. Ligier Y, Ratib O, Logean M, et al: Osiris: A medical image manipulation system. MD Computing Journal 11:212218, 1994

29. Wernecke J: The Inventor Mentor. Reading, MA, Addison-Wesley, 1994

30. Ames AL, Nadeau DR, Moreland JL: The VRML Source Book. New York, NY, Wiley, 1996

31. Gleason PL, Kikinis R, Altobelli D, et al: Video 
registration virtual reality for non linkage stereotactic surgery. Stereotact Funct Neurosurg 63:139-143, 1994

32. Turkington G, Jaszczak RJ, Pelizzari CA, et al: Accuracy of registration of PET, SPECT and MR images of brain phantom. J Nucl Med 34:1587-1594, 1993

33. Bidaut LM: Composite PET and MRI for accurate localization and metabolic modeling: A very useful tool for research and clinic. SPIE Medical Imaging V 1445:66-77, 1991

34. Gonzalez RC, Wintz P: Digital Image Processing. Reading, MA, Addison-Wesley, 1987

35. Barillot C: Surface and volume rendering techniques to display 3D data. IEEE Engineering in Medicine and Biology 12:111-119, 1993

36. Toga A (ed): Three-Dimensional Neuroimaging. New York, NY, Raven, 1990

37. Bidaut LM, Levivier M, Goldman S, et al: Multimodality stereotaxic correlation between XCT, PET, MRI and histoiogy for tumoral tissue evaluation in the brain. Proceedings of the 14th Annual International Conference of the IEEE-EMBS 1215-1216, 1992

38. Bookstein FL: Principal warps: Thin-plate splines and the decomposition of deformations. IEEE Trans Pattern Anal Mach Intell 11:567-585, 1989

39. Beier T, Neely S: Feature-based image metamorphosis. Comput Graph 26:35-40, 1992
40. Liang Z: Tissue classification and segmentation of MR images. IEEE Eng Med Biol 12:81-85, 1993

41. Wells WM, Grimson WEL, Kikinis R, et al: Adaptative segmentation of MRI data. Ayache $\mathrm{N}$ (ed): Computer Vision, Virtual Reality and Robotics in Medicine. New York, NY, Springer-Verlag, 1995, pp 59-69

42. Mangin J-F, Frouin V, Bloch I, et al: Automatic construction of an attributed relational graph representing the cortex topography using homotopic transformations. SPIE Mathematical Methods in Medical Imaging III 2299: 110-121, 1994

43. Sperling MR, Alavi A, Reivich $M$, et al: False lateralization of temporal lobe epilepsy with FDG positron emission tomography. Epilepsia 36:722-727, 1995

44. Gaillard WD, Fazilat S, White S, et al: Interictal metabolism and blood flow are uncoupled in temporal lobe cortex of patients with complex partial epilepsy. Neurology 45:1841-1847, 1995

45. Friston $\mathrm{KJ}$, Frith $\mathrm{CD}$, Liddle PF, et al: Comparing functional (PET) images: The assessment of significant change. J Cereb Blood Flow Metab 11:690-699, 1991

46. Verkindt C, Bertrand O, Perrin F, et al: Tonotopic organization of the human auditory cortex: N100 topography and multiple dipole model analysis. Electroencephalogr Clin Neurophysiol 96:143-156, 1995

\title{
New Digital Imaging Book Announcements
}

\author{
Picture Archiving and Communication Systems in Biomedical Imaging
}

\author{
by H.K. Huang, DSc
}

VCH Publishers, Inc.; 303 NW 12th Avenue; Deerfield Beach, FL 33442; Fax: 800-367-8247 Outside of North America, VCH Verlagsgesellschaft mbH; P.O. 10-11-61; 69451 Weinheim FRG.

$\$ 150.00$ plus handling. Call 800-961-8249. 489 pages.

\section{Imaging and Information Management: Computer Systems for a Changing Health Care Environment (May, 1996 Radiological Clinics of North America)}

\section{by Robert A. Greenes, MD, PhD, and Roger A. Bauman, MD}

W.B. Saunders Co.; Periodicals Marketing; Independence Square West; Philadelphia, PA 19106; Fax: 407-363-9661

$\$ 31.00$ (includes handling); add $\$ 6.00$ for airmail to Europe)

Call 800-654-2452. 248 pages. 